\title{
Human Factors-Based Many-Objective Personnel Recruitment for Safety-Critical Work Environments
}

\author{
Beatrice Lazzerini and Francesco Pistolesi \\ Department of Information Engineering - University of Pisa, Italy \\ Email: $\{$ b.lazzerini, f.pistolesi\}@iet.unipi.it
}

\begin{abstract}
In spite of many improvements in industrial safety of the last decades, nowadays four people per minute die in the world for occupational illnesses and accidents at work. Besides equipping machines with the most advanced technologies, industrial safety has become more and more interested in human factors in recent years, since many accidents at work are proven to be blamed on dangerous behaviours of workers. Recruiting workers with proper risk perception and caution can make safer their interaction with the task assigned, thus reducing devastating events. This paper presents a many-objective optimization framework for personnel recruitment in safety-critical work environments. Four objectives are considered: cost and learning time (which are minimized), and risk perception and caution (which are maximized). A neural network-based module computes each candidate's risk perception and caution for every single task he/she applies for. Pareto optimal solutions are generated using the Multi-Objective Particle Swarm Optimizer based on hypervolume (MOPSOhv). The best personnel recruitment is selected by the Technique for Order of Preference by Similarity to Ideal Solution (TOPSIS). The effectiveness of the proposed framework was validated on two real-world recruitment processes involving 100 and 300 candidates, respectively.
\end{abstract}

\section{INTRODUCTION}

Human factors have become increasingly important in recent years to improve the safety of work environments. This is due to the fact that, despite huge technological advances that have led to the design and construction of more and more sophisticated and secure machines, worldwide, every year, about 2.3 million people die due to occupational injuries (318,000 deaths) and work-related diseases (2,022,000 deaths): 4 people per minute [1]. It is estimated that up to $80 \%$ of the accidents at work are blamed on actions or omissions of workers [2].

Human factors refer to environmental, organizational and job factors, and individual characteristics which influence the behaviour at work in a way that can affect health and safety [2]. For instance, with respect to individual characteristics, people have their own personal attitudes, skills and personalities. These aspects can act as strengths or weaknesses, depending on the task performed, and they can cause dangerous behaviours. Individual characteristics affect people's behaviour in complex ways. Therefore, human factors influence risk perception, which is the way one realizes characteristics and gravity of dangerous situations [3]-[7]. Examples of human factors include past health status, past experience, cultural, social and psychological aspects, trust in risk management institutions, age, locus of control [8], [9] and optimism bias [9], [10]. Even though all these factors have been studied in depth, it is still unclear how they influence the behaviour in the presence of risk.

Risk awareness programs are aimed at making employees aware of the dangers, through safety training courses. These courses are iterated at regular intervals so as to let workers maintain a proper risk perception level to carry out the tasks safely. Nevertheless, many accidents happen every day. Recently, advanced techniques have been proposed to identify the workers' risk sensibility profiles [11], with the aim of classifying each worker into one of them [12], and provide him/her a customized training wherein concepts are presented in the most appropriate way. However, these techniques require significant economic resources. Just think, for instance, that occupational illnesses and accidents cause costs ranging between $1.8 \%$ and $6 \%$ of GDP, in country estimates, with an average of $4 \%$ [1]. The lack of economic resources makes it difficult for safety to be managed in small and medium enterprises (SMEs). In the European Union, SMEs represent 99\% of the companies and employ 65 million people [13].

Integrating human factors into industrial safety strategies can reduce accidents and occupational diseases [2]. Therefore, instead of repeating safety training courses (with the economic costs discussed above), it may be helpful to consider human factors during the recruitment of new personnel. So doing, companies could hire the most suitable person for each vacant job, from the point of view of safety. This can be achieved by considering, on the one hand, the characteristics of the job and its risks, and, on the other hand, the candidates' human factors, in order to determine their risk perception level and caution in the presence of risk.

This paper presents a framework for many-objective optimization in the field of personnel recruitment. Four objectives are considered, namely, the cost and the learning time of a new task (which are minimized), and the risk perception and caution (which are maximized). For each candidate, the learning time of a new task is estimated by analyzing his/her past jobs, based on a three-phase model of the learning curve. Each candidate's risk perception and caution are computed by a neural network-based system, by considering the candidates' human factors and the characteristics of their behaviour in the presence of risk. The optimization problem is solved in two steps. First, a hypervolume-based particle swarm multiobjective algorithm (i.e., MOPSOhv [14]) generates a set of Pareto optimal recruitment plans. Then, the best recruitment 
plan is selected by means of the TOPSIS algorithm. The proposed framework was tested on two real-world recruitment scenarios involving 100 and 300 candidates, respectively. By comparing the recruitment plans suggested by the management to those generated by our framework, significant increase in risk perception and caution is achieved, with modest increase in cost. This can actually make risks less likely and/or less damaging. Safety in the workplace can be significantly improved because workers are more aware of the dangers and therefore they are more cautious at work.

The paper is organized as follows: Section II contains some preliminaries on multi-criteria decision making and multi/many-objective optimization; in Section III the model of the worker's risk perception and caution is given; Section IV presents the problem formulation and the objective functions; in Section V the experiments are discussed and, in Section VI, the conclusions are drawn.

\section{PRELIMINARIES}

\section{A. Multi-criteria decision making}

A multi-criteria decision making (MCDM) problem has a set of criteria and a set of alternatives. Finding the best alternative with respect to all the criteria is the goal.

1) Technique for Order of Preference by Similarity to Ideal Solution (TOPSIS): TOPSIS is an MCDM methodology [15]. For an $n$-alternatives and $m$-criteria decision problem, TOPSIS needs an $n \times m$ decision matrix $\mathbf{H}=\left[h_{i j}\right]$, where $i \in\{1, \ldots, n\}$ and $j \in\{1, \ldots, m\}$. Element $h_{i j}$ measures how good alternative $i$ is with respect to criterion $j$. Also, criteria are required to be weighted. Let $\boldsymbol{\omega}=\left(\omega_{1}, \ldots, \omega_{m}\right)$, with $\sum_{j=1}^{m} \omega_{j}=1$, contain the weights of the criteria. TOPSIS computes the weighted normalized decision matrix $\mathbf{V}=\left[v_{i j}\right]=\omega_{j} h_{i j} / \sqrt{\sum_{i=1}^{n} h_{i j}^{2}}$. The ideal best $(I B)$ and worst $(I W)$ solutions are then found. Let benefit and cost criteria be indexed by indices in $\Omega_{B}$ and $\Omega_{C}$, respectively. Let $I B=\left(a_{1}^{+}, \ldots, a_{m}^{+}\right)$and $I W=\left(a_{1}^{-}, \ldots, a_{m}^{-}\right)$, where $a_{j}^{+}=\max _{i} v_{i j}$ for $j \in \Omega_{B}$ or $a_{j}^{+}=\min _{i} v_{i j}$ for $j \in \Omega_{C}$, and $a_{j}^{-}=\min _{i} v_{i j}$ for $j \in \Omega_{B}$ or $a_{j}^{-}=\max _{i} v_{i j}$ for $j \in \Omega_{C}$. TOPSIS computes the Euclidean distance of each alternative from $I B$, i.e., $D_{i}^{+}=\sqrt{\sum_{j=1}^{m}\left(v_{i j}-a_{j}^{+}\right)^{2}}$, and $I W$, i.e., $D_{i}^{-}=\sqrt{\sum_{j=1}^{m}\left(v_{i j}-a_{j}^{-}\right)^{2}}$. Finally, TOPSIS determines the relative closeness coefficient of each alternative to $I B$ as $R C L_{i}^{+}=D_{i}^{-} /\left(D_{i}^{+}+D_{i}^{-}\right)$: the higher $R C L_{i}^{+}$the better. Alternative $k=\arg \max _{i} R C L_{i}^{+}$is selected.

\section{B. Multi- and Many-objective optimization}

A multi-objective optimization (MOO) problem [16] involves the optimization of two or more (conflicting) objectives. The general MOO problem form is

$$
\underset{\mathbf{x}}{\operatorname{Minimize}} \mathbf{f}(\mathbf{x})=\left[f_{1}(\mathbf{x}), f_{2}(\mathbf{x}), \ldots, f_{k}(\mathbf{x})\right]
$$

subject to:

$$
\begin{aligned}
& g_{i}(\mathbf{x}) \leq 0, \quad i=1, \ldots, m \\
& h_{j}(\mathbf{x})=0, \quad j=1, \ldots, n
\end{aligned}
$$

where $k \geq 2$ is the number of objectives, while $m$ and $n$ are the number of inequality and equality constraints, respectively. Function $\mathbf{f}: \mathbb{R}^{p} \rightarrow \mathbb{R}^{k}$ contains the objective functions to minimize. In general, no solution exists which minimizes all the objective functions. This is why Pareto dominance and Pareto-optimality are considered. A solution $\mathbf{x}^{\mathbf{1}}$ is said to dominate $\mathbf{x}^{2}$ if $f_{i}\left(\mathbf{x}^{\mathbf{1}}\right) \leq f_{i}\left(\mathbf{x}^{2}\right) \forall i \in\{1, \ldots, k\}$, and $f_{j}\left(\mathbf{x}^{\mathbf{1}}\right)<f_{j}\left(\mathbf{x}^{\mathbf{2}}\right)$ for at least one index $j \in\{1, \ldots, k\}$. Pareto-optimal solutions cannot be improved with respect to any objective without degrading at least one of the remaining.

Many-Objective Optimization (MaOO) deals with optimization problems having more than 3 objectives [17].

1) Particle swarm optimization: Particle Swarm Optimization (PSO) is an optimization heuristics inspired by the social behaviour of birds flocking to find food [18]. Given an optimization problem, the set of solutions is represented by a set of particles (i.e., the swarm) moving through the search space, according to a cooperative procedure. In PSO, the swarm searches for optimal solutions through generations. The movement of each particle depends on the velocity operator, which is controlled by a local and a social component. In particular, the behaviour of a particle is influenced by either the best personal (i.e., within a neighborhood) or the best global particle (i.e., the leader). Personal particles and leaders represent the local and social components, respectively.

Multi-Objective Particle Swarm Optimization (MOPSO) [19] typically uses Pareto dominance to determine leaders. Using Pareto dominance, each particle may have more than one leader, but only one is selected to update the velocity. This set of leaders is stored into an external archive containing the best non-dominated solutions found so far.

The steps of a MOPSO algorithm are: i) initialization of the swarm; ii) computation of the velocity; iii) position update and archive update. Each particle $p_{i}$ has a position $\mathbf{x}_{\mathbf{i}}(t) \in \mathbb{R}^{p}$ and a velocity $\mathbf{v}_{\mathbf{i}}(t) \in \mathbb{R}^{p}$ at time $t$. Position and velocity are updated as follows:

$$
\begin{gathered}
\mathbf{x}_{\mathbf{i}}(t+1)=\mathbf{x}_{\mathbf{i}}(t)+\mathbf{v}_{\mathbf{i}}(t+1) \\
\mathbf{v}_{\mathbf{i}}(t+1)=\omega \mathbf{v}_{\mathbf{i}}(t)+C_{1} r_{1}\left(\mathbf{x}_{p_{i}}-\mathbf{x}_{i}\right)+C_{2} r_{2}\left(\mathbf{x}_{g_{i}}-\mathbf{x}_{i}\right)
\end{gathered}
$$

where $\mathbf{x}_{p_{i}}$ is the best solution (position) ever explored by particle $\mathbf{x}_{i}, \mathbf{x}_{g_{i}}$ is the leader, i.e., the best solution the entire swarm has encountered. Also, $\omega$ is the inertia, which determines how the particle velocity is influenced by the previous velocity. Finally, $C_{1}$ and $C_{2}$ control the effect of the best personal and global particles, respectively, and $r_{1}$ and $r_{2}$ are random numbers with uniform probability in $[0,1]$.

2) The MOPSOhv algorithm: MOPSOhv is a hypervolumebased multi-objective particle swarm algorithm. MOPSOhv proved to be effective in many-objective optimization problems [14]. From an operation point of view, MOPSOhv randomly initializes the swarm with $N$ particles. Positions and velocities are evaluated with respect to the $k$ objective functions. Considering the initial swarm, non-dominated solutions are inserted into the initial archive $A_{0}$. Then, in the main loop, the hypervolume contribution of each particle contained 
in the archive is first determined. Such particles are sorted in descending order, considering their hypervolume contribution. For each particle, global and personal leaders are determined. The global leader is chosen from the top-ranked particles (from the top 2\%) of the sorted archive $A_{t}$. The personal leader is chosen from the lowest-ranked particles (the bottom 98\%). Each particle in the swarm updates its velocity and position according to the leaders. The new position of each particle must be in the search space. So, if the position $\mathbf{x}_{\mathbf{i}}(t+1)$ of particle $i$ at time $t+1$ goes beyond a boundary of some decision variable, particle $i$ is put back into the search space, by assigning the corresponding lower or upper bound to the decision variable. Also, its velocity is inverted. In the last step of the update, a mutation operator is applied to each particle, based on a mutation probability that depends on time $t$. Soupdated particles are evaluated with respect to the objectives of the problem, and the new non-dominated particles are selected to construct archive $A_{t+1}$. In this final step, new solutions might dominate some solutions in the archive. In such a case, the latter are deleted. Also, new solutions may be non-dominated with respect to the entire archive, hence, new non-dominated solutions are inserted into the archive, which is then pruned to its maximum size by deleting those particles that contribute the least to the hypervolume.

\section{WORKER'S RISK PERCEPTION AND CAUTION}

Consider a work environment with a set of tasks $\mathcal{T}$. Let $\mathcal{W}=\left\{w_{1}, \ldots, w_{|\mathcal{W}|}\right\}$ be the set of the workers. Task $t_{i}$, with $i=\{1, \ldots,|\mathcal{T}|\}$, exposes a worker to a set $\mathcal{R}_{i}$ of risks. The set $\mathcal{R}=\bigcup_{i=1}^{|\mathcal{T}|} \mathcal{R}_{i}$ contains all the risks of the workplace.

A set $\mathcal{A}_{k}=\left\{a_{k, 1}, \ldots, a_{k,\left|\mathcal{A}_{k}\right|}\right\}$ of preventive actions can be used by workers to prevent each risk $r_{k} \in \mathcal{R}$, with $k \in\{1, \ldots,|\mathcal{R}|\}$. A preventive action can make a risk less likely and/or decrease its effects, the so-called risk impact. A prevention level in $\mathcal{L}=\{1, \ldots, L\}$ characterizes each preventive action. The higher the level the more effective the action in decreasing probability and/or impact of a risk. Typically, experts in risk assessment establish the prevention level of an action.

Let $\mathcal{H}=\left\{h_{1}, \ldots, h_{|\mathcal{H}|}\right\}$ be a set of human factors (hereafter, factors). Each factor $h_{v}$, where $v \in\{1, \ldots,|\mathcal{H}|\}$, takes values in a domain $\mathcal{D}_{v}$. Two categories of factors form set $\mathcal{H}$, the ones relating to the individual and the ones relating to the task. The former are $P$ personal factors, while the latter are $T$ task-related factors. For worker $w_{j}$, where $j \in$ $\{1, \ldots,|\mathcal{W}|\}$, the risk perception personal level pers_perc $c_{j}$ depends on the set $\mathcal{P}_{j}=\bigcup_{v=1}^{P} d_{v, j}$, where $d_{v, j} \in \mathcal{D}_{v}$ is the value of personal factor $h_{v}$ for worker $w_{j}$. Obviously, $\mathcal{P}_{j} \in \mathcal{D}_{1} \times \cdots \times \mathcal{D}_{P}$. A function $\varphi_{P E R S O N A L}$ exists, such that $\mathcal{P}_{j} \mapsto \varphi_{P E R S O N A L}\left(\mathcal{P}_{j}\right)=$ pers_perc ${ }_{j}$.

The perception level task $\_p e r c_{i, j}$ of $w_{j}$ for the risks of task $t_{i}$ stems from $\mathcal{T}_{j}=\bigcup_{v=P+1}^{P+T} d_{v, j}$. Here, $d_{v, j}$ is the value of task-related factor $h_{v}$ for worker $w_{j}$. Also, task_perc $_{i, j}$ depends on the risk perception personal level pers_perc $c_{j}$ of $w_{j}$. Hence, there exists function $\varphi_{T A S K}$ such that $\left(\mathcal{T}_{j}\right.$, pers_perc $\left.c_{j}\right) \mapsto \varphi_{T A S K}\left(\mathcal{T}_{j}\right.$, pers_perc $\left._{j}\right)=$ task_perc $c_{i, j}$.

For each risk $r_{k}$ and worker $w_{j}$, the caution of $w_{j}$ for $r_{k}$ stems from the count of preventive actions $w_{j}$ performs to prevent $r_{k}$, for each prevention level: this represents the behaviour of $w_{j}$ toward $r_{k}$. More formally, let $\# \mathcal{A}_{k, \ell=\bar{\ell}, j}$ denote the count of $\bar{\ell}$-level actions $w_{j}$ performs to prevent $r_{k}$. A function $\rho_{k}$, such that $\left(\# \mathcal{A}_{k, \ell=1, j}, \ldots, \# \mathcal{A}_{k, \ell=L, j}\right) \mapsto$ $\rho_{k}\left(\# \mathcal{A}_{k, \ell=1, j}, \ldots, \# \mathcal{A}_{k, \ell=L, j}\right)=$ risk_caution $_{k, j}$, exists for each $k=1, \ldots,|\mathcal{R}|$.

Finally, for each task $t_{i}$ and worker $w_{j}$, the caution of $w_{j}$ for $t_{i}$ depends on risk_caution $k, j, \forall k \in \mathcal{R}_{i}$. A set of functions $\tau_{i}$, one for each $i=1, \ldots,|\mathcal{T}|$, such that $\bigcup_{r_{k} \in \mathcal{R}_{i}}$ risk_caution $_{k, j} \mapsto \tau_{i}\left(\bigcup_{r_{k} \in \mathcal{R}_{i}}\right.$ risk_caution $\left.k_{k, j}\right)=$ task_caution $_{i, j}$, computes the task caution of $w_{j}$ for each task $t_{i}$ given the worker's risk cautions.

In this model, worker $w_{j}$ is represented by the tuple

$$
\theta_{j}=\left\{\bigcup_{v=1}^{P+T} d_{v, j}, \bigcup_{k=1}^{|\mathcal{R}|} \bigcup_{\lambda=1}^{L} \# \mathcal{A}_{k, l=\lambda, j}\right\} .
$$

Note that $|\mathcal{H}|=P+T$ and $v \in\{1, \ldots, P\}$ denotes personal factors, while task-related factors are $v \in\{P+1, \ldots, P+T\}$.

A neural system was proposed in [20] to compute task_perc $c_{i, j}$ and task_caution ${ }_{i, j}$, given the tuple in Eq. (6), which describes worker $w_{j}$ and how he/she interacts with each task $t_{i}$. This system is used in this paper to obtain the risk perception and caution of a candidate with respect to each task he/she applies for. These values determine two objectives of the optimization problem formalized in the next section.

\section{OPTIMIZATION PROBLEM}

\section{A. Objective functions}

Let $x_{i j} \in\{0,1\}$ be a decision variable such that

$$
x_{i j}= \begin{cases}1 & \text { if worker } w_{j} \text { is recruited for task } t_{i} \\ 0 & \text { otherwise }\end{cases}
$$

where $i \in\{1, \ldots,|\mathcal{T}|\}$ and $j \in\{1, \ldots,|\mathcal{W}|\}$. Vector $\mathbf{x} \in\{0,1\}^{|\mathcal{T}| \times|\mathcal{W}|}$ represents a personnel recruitment plan, and contains variables $x_{i j}$ in lexicographic order. The four objectives taken into account in this paper are formalized in the following.

1) Cost: The cost of assigning task $t_{i}$ to worker $w_{j}$ depends on his/her work seniority for $t_{i}$. The greater the work seniority for $t_{i}$, the higher the cost $c_{i j}$ the employer pays (for salary and benefits) to assign worker $w_{j}$ to task $t_{i}$. The global cost objective function $\operatorname{COST}(\mathbf{x}):\{0,1\}^{|\mathcal{T}| \times|\mathcal{W}|} \rightarrow \mathbb{R}^{+}$, here minimized, is modeled as:

$$
\operatorname{COST}(\mathbf{x})=\sum_{i=1}^{|\mathcal{T}|} \sum_{j=1}^{|\mathcal{W}|} c_{i j} x_{i j} .
$$

2) Learning time: In the model here proposed, more experienced workers are preferred to be recruited for each task. To be correctly performed, a task $t_{i}$ requires a set of skills $\mathcal{S}_{i}$. Consider worker $w_{j}$ applying for task $t_{i}$. Let $\mathcal{P}_{j}$ be the set of the past jobs of $w_{j}$ and let $D_{p_{j}}^{S T A R T}$ and $D_{p_{j}}^{E N D}$ be the dates on which $w_{j}$ began and ceased past job $p_{j} \in \mathcal{P}_{j}$, respectively. 
Also, let $t\left(p_{j}\right)$ be the task performed by $w_{j}$ in $p_{j}$. The set $\mathcal{P}_{i, j}^{S K I L L}=\left\{p_{j} \in \mathcal{P}_{j}: \mathcal{S}_{i} \cap \mathcal{S}_{t\left(p_{j}\right)} \neq \emptyset\right\}$ contains the past jobs of worker $w_{j}$ which required some skills of task $t_{i}$.

In this paper, with reference to a past job in which worker $w_{j}$ performed task $t_{i}$, a learning curve like the one shown in Fig. 1 is considered. In particular, the curve is composed of

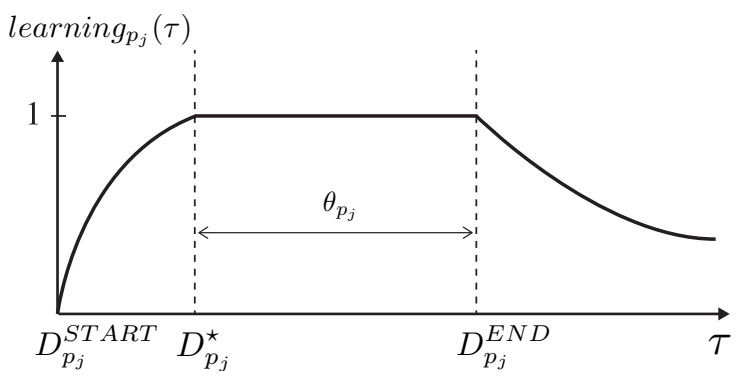

Fig. 1. Learning curve of worker $w_{j}$ in past job $p_{j}$.

three pieces. The first one corresponds to the learning phase, wherein the worker has to be trained to perform the task. The learning process requires a time for the worker to learn how to perform the task properly. The duration of the learning phase is measured from $D_{p_{j}}^{S T A R T}$ to the day when the worker is considered to be sufficiently skilled for the task. This happens at time $D_{p_{j}}^{\star}$, when the worker can execute the task repeatedly, completing its steps in a certain time and with a given quality level. From $D_{p_{j}}^{\star}$ to $D_{p_{j}}^{E N D}$, the worker's skill is considered unchanged because the task is performed every day. When the worker ceases a job, generally tends to forget the details of the task and how to perform it, as time passes. The model here considered is as follows:

$$
\begin{aligned}
& \text { learning }_{p_{j}}(\tau)= \\
& \left\{\begin{array}{ccc}
\lambda_{t\left(p_{j}\right)} \ln \left(\tau+D_{p_{j}}^{S T A R T}\right) & \text { if } & D_{p_{j}}^{S T A R T} \leq \tau \leq D_{p_{j}}^{\star} \\
1 & \text { if } & D_{p_{j}}^{\star}<\tau \leq D_{p_{j}}^{E N D} \\
e^{-\frac{1}{\ln \theta p_{j}} \tau+B} & \text { if } & \tau>D_{p_{j}}^{E N D}
\end{array}\right.
\end{aligned}
$$

where $\tau$ is the time in days, $B=\frac{D_{p_{j}}^{E N D}}{\ln \theta_{p_{j}}}$, and $\theta_{p_{j}}$ is the count of the days with learning equal to 1 , that is, the central region of Fig. 1. In order to recruit a worker $w_{j}$ for a task $t_{i}$, it is possible to estimate how long the time to learn the new task will be. Once set $\mathcal{P}_{i, j}^{S K I L L}$ is determined, for each $p_{j} \in$ $\mathcal{P}_{i, j}^{S K I L L}$ it holds that

$$
\lambda_{t\left(p_{j}\right)}=\frac{1}{D_{p_{j}}^{\star}+D_{p_{j}}^{S T A R T}} .
$$

Therefore, for a candidate $w_{j}$ applying for task $t_{i}$, we estimate the coefficient $\lambda_{i, j}$ affecting the learning time of $w_{j}$ as

$$
\lambda_{i, j}=\frac{1}{\left|\mathcal{P}_{i, j}^{S K I L L}\right|} \sum_{p_{j} \in \mathcal{P}_{i, j}^{S K I L L}} \lambda_{t\left(p_{j}\right)}\left(\frac{\left|\mathcal{S}_{t\left(p_{j}\right)}\right|}{\left|\mathcal{S}_{i}\right|}\right),
$$

that is, a weighted average of the $\lambda_{t\left(p_{j}\right)}$ where the more the skills required by past job $p_{j}$ also required by the task the candidate applies for (i.e., $t_{i}$ ), the higher the weight. By considering the learning curve in Eq. (19) with coefficient $\lambda_{i, j}$ in place of $\lambda_{t\left(p_{j}\right)}$, one can get the time $D_{i, j}^{\star}$ required to train worker $w_{j}$ for task $t_{i}$, based on the estimate in Eq. (21), as

$$
D_{i, j}^{\star}=\frac{1-\lambda_{i, j} D_{i, j}^{S T A R T}}{\lambda_{i, j}}
$$

where $D_{i, j}^{S T A R T}$ is the date when worker $w_{j}$ begins the new job where he/she performs task $t_{i}$. The learning time objective

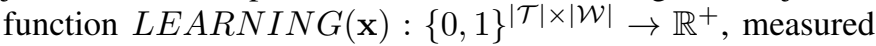
in days, is here defined as:

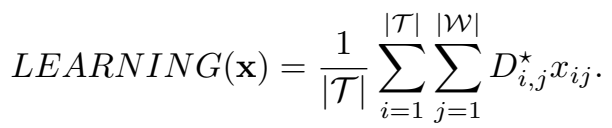

The average is used because learning times are supposed to be quite similar. The global learning time is minimized.

3) Perception: Consider the average perception level of a personnel recruitment plan $\mathbf{x}$ expressed as: $\overline{\text { perc }}=\frac{1}{|\mathcal{T}|} \sum_{i=1}^{|\mathcal{T}|} \sum_{j=1}^{|\mathcal{W}|}$ task_perc $_{i, j} x_{i j}$. Global risk perception $\operatorname{PERC}(\mathbf{x}):\{0,1\}^{|\mathcal{T}| \times|\mathcal{W}|} \rightarrow \mathbb{R}^{+}$, to be maximized, is measured here as the mean to variance ratio of the risk perception of every worker with respect to the task assigned:

$$
\operatorname{PERC}(\mathbf{x})=\frac{\overline{p e r c}}{\sum_{i=1}^{|\mathcal{T}|} \sum_{j=1}^{|\mathcal{W}|}\left(\text { task_perc } c_{i, j} x_{i j}-\overline{p e r c}\right)^{2}} .
$$

4) Caution: Consider the average task caution of a personnel recruitment plan $\mathbf{x}$ expressed as: $\overline{\text { caution }}^{=} \frac{1}{|\mathcal{T}|} \sum_{i=1}^{|\mathcal{T}|} \sum_{j=1}^{|\mathcal{W}|}$ task_caution $_{i, j} x_{i j}$. Global caution $\operatorname{CAUTION}(\mathbf{x}):\{0,1\}^{|\mathcal{T}| \times|\mathcal{W}|} \rightarrow \mathbb{R}^{+}$is modelled here as the mean to variance ratio of the caution of every worker with respect to the task assigned:

$\operatorname{CAUTION}(\mathbf{x})=\frac{\overline{\text { caution }}}{\sum_{i=1}^{|\mathcal{T}|} \sum_{j=1}^{|\mathcal{W}|}\left(\text { caution }_{i, j} x_{i j}-\overline{\text { caution }}\right)^{2}}$.

Global caution is maximized.

\section{B. Problem formulation}

Consider a set of tasks $\mathcal{T}$ and a set of workers $\mathcal{W}$ where $|\mathcal{W}| \lesseqgtr|\mathcal{T}|$. Assume that each task can be assigned to one worker and vice versa. The optimization problem is:

$$
\begin{array}{cc}
\underset{\mathbf{x}}{\operatorname{Minimize}} & \mathbf{f}(\mathbf{x})=[\operatorname{COST}(\mathbf{x}), \operatorname{LEARNING}(\mathbf{x}), \\
-\operatorname{PERC}(\mathbf{x}),-\operatorname{CAUTION}(\mathbf{x})]
\end{array}
$$

subject to:

$$
\begin{gathered}
\sum_{i=1}^{|\mathcal{T}|} x_{i j} \leq 1, \quad \forall j=1, \ldots,|\mathcal{W}| \\
\sum_{j=1}^{|\mathcal{W}|} x_{i j}=1, \quad \forall i=1, \ldots,|\mathcal{T}| \\
x_{i j} \in\{0,1\}, \quad \forall i=1, \ldots,|\mathcal{T}|, \forall j=1, \ldots,|\mathcal{W}| .
\end{gathered}
$$


Equation (26a) is the vector-valued objective function $\mathbf{f}(\mathbf{x})$ : $\{0,1\}^{|\mathcal{T}| \times|\mathcal{W}|} \rightarrow \mathbb{R}_{+} \times \mathbb{R}_{-}^{3}$ whose components are, in the order, the global cost, the learning time, the global risk perception, and the global caution for recruitment $\mathbf{x} \in\{0,1\}^{|\mathcal{T}| \times|\mathcal{W}|}$. Constraints (26b) consider a worker may be assigned to no task, since workers and tasks are typically unbalanced in recruitment problems. Constraints (26c) let each task be assigned to one worker. Finally, Eq. (26d) is the integer constraint.

\section{EXPERIMENTS AND DISCUSSION}

This section describes the results obtained by applying the proposed framework to two real-world recruitment scenarios, hereafter referred to as REC1 and REC2, respectively. Experiments were carried out thanks to two footwear companies.

To the best of the authors' knowledge, there is no previous work in the literature which deals with cost, learning time, risk perception and caution in the personnel recruitment problem. For this reason no comparison with previous works could be made. Instead, results were compared to the recruitment plans suggested by the management of the involved companies.

Also, it is important to point out that risk perception and caution are completely new measures and they are hard to be evaluated objectively because there is no ground truth to be used for this. However, a way to evaluate the results may be thinking of how safely workers say they would behave in the presence of risk, by considering the level of prevention of the selected preventive actions, in intuitive terms.

\section{A. Dataset and data gathering}

The optimization framework was implemented in Java and MATLAB. Also, a website containing a multiple choice test was implemented in Java EE and MySQL. Once deployed on a web server, the website allowed candidates of the two experimented scenarios to fill out a questionnaire. Data were gathered anonymously, in compliance with the privacy laws. For each candidate $w_{j}$, the questionnaire collects:

- data about the candidate's human factors to determine task_perc $c_{i, j}$;

- data related to the candidate's past jobs to derive the learning time for each task the candidate applies for;

- data related to the behaviour towards each risk $r_{k}$ of every single task $t_{i}$ the candidate applies for, i.e., the preventive actions the candidate selects from a set of proposed preventive actions. These selected actions let the system compute $t_{a s k}$ caution $_{i, j}$ for each task $t_{i}$ the candidate applies for.

For lack of space, the questionnaire cannot be reported here.

The collected dataset consists of 400 interviews. A first set of 100 interviews is related to candidates of the recruitment process carried out by the first company. The remaining 300 interviews concern candidates of the recruitment process of the other company. Results are discussed in the next section.

\section{B. Optimization results}

1) Personnel recruitment in the involved companies: Companies carry out personnel recruitment processes whenever new employees have to be hired for vacant tasks. In the involved companies, experts determine how experienced each candidate is in performing a task by means of risk-free practical and technical tests. Several days are generally required for these tests to be carried out. Finally, candidates face an oral interview.

Based on the outcome of the practical tests and the interview, a suitability level is determined for each candidate with respect to every single position (i.e., task) he/she applies for. In addition, cost is taken into account. Assigning a candidate to a task is much more expensive the lower he/she is experienced for that task and the higher the candidate's work seniority.

The great majority of the tasks of a footwear industry consists of handmade work. In the remaining tasks, workers use (potentially dangerous) machines, in a repetitive way, to cut the leather, scrape the soles, sew the uppers and so on. The risks to which workers are exposed include crushing, amputation, burn, and fall. Nevertheless, risk management is often carried out in SMEs by assigning more dangerous tasks to candidates with more years of experience, regardless of their human factors and behaviour in the presence of risk. This may be extremely dangerous because more experienced workers get higher locus of control and this can reduce risk perception [8].

2) Proposed technique for personnel recruitment: The management of the involved company was first asked to express the weights of the objectives (see Table III). An expert in risk assessment classified the preventive actions into three prevention levels (i.e., low, medium, high).

Data about each candidate's human factors and behaviour were gathered through the website described in Section V-A. Such data let us obtain each candidate's task_perc and risk_caution toward each risk. The task_caution and the task_perc (with respect to the tasks each candidate applies for) were first computed by the neural system referred to in Section III. The Pareto front was approximated by means of MOPSOhv with the parameters in Table IV. Parameters of Table IV produced the best results and have been statistically validated with the paired $t$-Student's test, by considering different configurations. For each tested configuration, the average hypervolume of 20 simulations was computed. The best solution, selected by TOPSIS, is in Table V.

3) Discussion of RECl: Within REC1, the company had 10 vacant tasks. The management gave 100 candidates the possibility to apply. The aim here is to improve safety without particular constraints on cost and learning time. As Table III shows, the sum of the weights associated with risk perception and caution is more than twice the sum of the weights of cost and learning. The obtained Pareto front is shown in Fig. 2.

The management suggested a recruitment plan having global risk perception and global caution equal to -3.37 and -4.03 , respectively, as shown in Table $\mathrm{V}$. The proposed recruitment guarantees $\sim 105 \%$ improvement in global risk perception and $\sim 94 \%$ improvement in global caution.

Since global risk perception and caution are difficult to evaluate, it is important to discuss some reasons the improvement in safety arises from. Data on the candidates' behaviour 
TABLE III

WEIGHTS OF THE OBJECTIVES

\begin{tabular}{|c|c|c|c|c|}
\cline { 2 - 5 } \multicolumn{1}{c|}{} & COST & LEARNING & PERC & CAUTION \\
\hline REC1 & 0.1 & 0.2 & 0.35 & 0.35 \\
\hline REC2 & 0.3 & 0.3 & 0.2 & 0.2 \\
\hline
\end{tabular}

TABLE IV

PARAMETERS USED FOR REC1 AND REC2

\begin{tabular}{|c|c|c|}
\cline { 2 - 3 } \multicolumn{1}{c|}{} & REC1 & REC2 \\
\hline Number of particles & 200 & 300 \\
\hline C1 & \multicolumn{2}{|c|}{1} \\
\hline C2 & \multicolumn{2}{|c|}{1} \\
\hline Mutation probability & \multicolumn{2}{|c|}{0.5} \\
\hline $\boldsymbol{\omega}$ & 0.4 & 0.5 \\
\hline Max iterations & \multicolumn{2}{|c|}{1500} \\
\hline
\end{tabular}

with respect to every single task cannot be reported here for reasons of space. Indeed, each job exposes workers to 5 risks on average, in the experimented scenarios. In turn, each risk can be prevented, on average, by 4 actions per prevention level. The proposed framework considers 3 prevention levels, low, medium and high. Therefore, considering for instance REC1 (the smaller scenario), on average there would be 60 preventive actions for worker, with a total of 1200 preventive actions, i.e., 600 related to the assignment suggested by management and 600 related to the assignment proposed by the framework. Thus, the discussion is here based on intuitive considerations about the behaviours that deeply impact on safety.

To understand the safety improvement that the proposed framework guarantees in REC1, think that if the assignment proposed were applied, just under two thirds of the workers would deal with the assigned task only with high-level preventive actions. The remaining one third of the workers would be characterized by behaviors ranging from a poor level of caution which is characterized by 2 low-level actions, to a good level of caution given by 3 medium-level actions and 1 high-level action. It is important to note that the most unsafe behaviour (i.e., 2 low-level actions only) stems from workers which stamp the shoe insoles or put the tissue paper into the shoes. As one can imagine, tasks like these do not expose workers to dangerous risks. In particular, workers may experience eyestrain, back pain and muscle tightness.

Instead, in the recruitment plan suggested by the management, 3 safety-critical tasks are assigned to workers with behaviour made of only 2 low-level preventive actions. These tasks deal with sander machines, high temperature ovens and diecutters. These machines can cause serious consequences on the health if used unsafely, because workers may experience severe grazes, burns and crushing of hands, and, in the case of the diecutters, even amputation. The workers that the management assigns to these tasks have a high probability of getting hurt because of their poor level of caution. The management, in these cases, makes the mistake of assigning safety-critical tasks to people having a greater number of years of experience. Of course, the management focuses on the fact that these workers do not need to be trained to perform the task properly, but it is highly wrong to neglect their behaviour in the presence of risk. Here, the workers assigned to the safetycritical tasks discussed so far have 8 years of experience for the assigned task, on average, but their level of caution is extremely poor, mainly because they have become familiar with the tasks and do not realize the risks they take with their behaviour. With reference to the learning time, the proposed recruitment is able to guarantee a faster training ( $\sim 7$ days, on average) if compared to the recruitment suggested by the management ( $\sim 10$ days, on average). The improvement in risk perception, caution and learning time discussed so far is achieved at the expense of an increase in cost of 3920 Euros. However, cost is not one of the most important objectives here (see Table III). Also, one should remember that cost decreases over time because it also considers the cost for the training.

4) Discussion of REC2: In REC2, cost and learning time are relevant, as shown in Table III. Hence, the management is here interested in recruitment plans characterized by a good level of risk perception and caution, but paying particular attention to cost and learning time.

The management suggested a plan with global risk perception and global caution equal to -5.29 and -6.48 , respectively (see Table V). With our plan, $550 \%$ improvement in global risk perception and $\sim 31 \%$ in global caution would be reached. The Pareto front is in Fig. 2. As for REC1, the discussion is based on the behaviours that highly impact on safety.

As an example, it is important to note that behaviours composed of only high-level preventive actions pass from 2 (in the suggested recruitment plan) to 5 (in the proposed recruitment plan). Also, thanks to our framework, no worker would have a behaviour composed of low-level preventive action only. Instead, in the recruitment suggested by the management, 4 behaviours like these exist, the riskiest ones are related, respectively, to a task where workers may crush

TABLE V

RECRUITMENT PLANS SUGGESTED BY THE MANAGEMENT (S) AND PROPOSED BY THE FRAMEWORK (P) WITH THEIR OBJECTIVES VALUES

\begin{tabular}{|c|c|c|c|c|c|c|c|c|c|c|c|c|c|c|c|c|c|c|c|c|c|c|c|c|c|}
\hline & \multicolumn{20}{|c|}{ TASKS } & \multicolumn{4}{|c|}{ OBJECTIVES } \\
\hline & & 1 & 2 & $\mathbf{3}$ & 4 & 5 & 6 & 7 & 8 & 9 & 10 & 11 & 12 & 13 & 14 & 15 & 16 & 17 & 18 & 19 & 20 & COST & LEARNING & $-P E R C$ & -CAUTION \\
\hline \multirow{2}{*}{ REC1 } & $\mathbf{S}$ & 94 & 71 & 19 & 38 & 90 & 44 & 66 & 13 & 84 & 7 & - & - & - & - & - & - & - & - & - & - & 26730 & 10.34 & -3.37 & -4.03 \\
\hline & $\mathbf{P}$ & 11 & 38 & 67 & 10 & 87 & 51 & 90 & 16 & 7 & 40 & - & - & - & - & - & - & - & - & - & - & 30650 & 7.28 & -6.91 & -7.84 \\
\hline \multirow{2}{*}{ REC2 } & $\mathbf{S}$ & 186 & 209 & 75 & 264 & 17 & 280 & 79 & 178 & 60 & 41 & 199 & 21 & 88 & 287 & 13 & 160 & 94 & 226 & 281 & 6 & 72993 & 12.38 & -5.29 & -6.48 \\
\hline & $\mathbf{P}$ & 138 & 86 & 19 & 244 & 280 & 97 & 209 & 178 & 110 & 60 & \begin{tabular}{|l|}
94 \\
\end{tabular} & 6 & 88 & 30 & 13 & 293 & 47 & 264 & 22 & 3 & 74160 & 13.06 & -7.93 & -8.51 \\
\hline
\end{tabular}



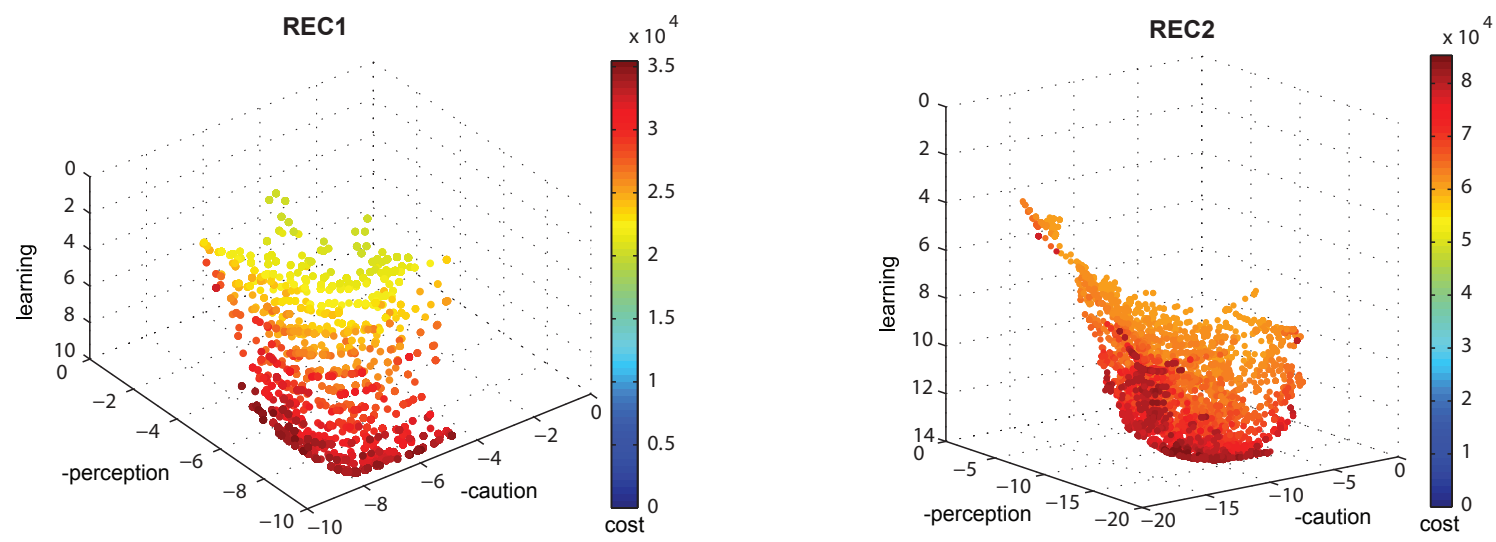

Fig. 2. Pareto fronts of REC1 and REC2. Circles represent Pareto-optimal recruitments.

their hands while using a press and to a task where workers may experience severe and irreversible burns to the hands. As in REC1, the recruitment proposed by the management is affected by the experience bias, i.e., the management chooses workers with more experience for the most dangerous tasks.

The learning time is almost the same as the recruitment suggested by the management (see Table V), and this respects the importance assigned by the management (see Table III).

Finally, it is important to highlight that the discussed increase in safety causes just $1.6 \%$ increase in global cost. This represents an unquestionable advantage of the proposed framework, especially in work environments related to SMEs.

\section{CONCLUSION}

This paper presented a many-objective optimization framework for personnel recruitment in safety-critical work environments. Four objectives are considered: the cost and the learning time are minimized, while the workers' risk perception and caution are maximized. Given a set of candidates, a neural system first computes each worker's risk perception and caution for every single task each candidate applies for. Pareto optimal solutions are then generated by using MOPSOhv, a hypervolume-based particle swarm multi-objective algorithm. The best personnel recruitment is finally selected by TOPSIS.

The proposed framework was tested on two real-world recruitment processes involving 100 and 300 candidates, respectively. Significant improvement of risk perception and caution has been shown in both scenarios by comparing the recruitment plan suggested by the management to the one found by the proposed framework. Risks are made less likely and less damaging because each vacant task is assigned to the most appropriate candidate from the point of view of safety. Also, modest increase in cost characterizes the proposed solutions. This is paramount for SMEs, a widely common reality in the EU.

\section{ACKNOWLEDGMENT}

This work was supported by the PRA 2016 project "Analisi di dati sensoriali: dai sensori tradizionali ai sensori sociali", funded by the University of Pisa.

\section{REFERENCES}

[1] http://www.ilo.org. International Labour Organization Statistics Database (ILOSTAT).

[2] Health and Safety Executive, Reducing error and influencing behaviour. HSE Books, 1999.

[3] M. Bouyer et al., "Personality correlates of risk perception," Risk Analysis, vol. 21, no. 3, pp. 457-465, 2001.

[4] B. Chauvin, D. Hermand, and E. Mullet, "Risk perception and personality facets," Risk Analysis, vol. 27, no. 1, pp. 171-185, 2007.

[5] E. Peters and P. Slovic, "The role of affect and worldviews as orienting dispositions in the perception and acceptance of nuclear power," Journal of applied social psychology, vol. 26, pp. 1427-1428, 1996.

[6] L. Sjöberg, "Factors in risk perception," Risk Analysis, vol. 20, no. 1, 2000.

[7] P. Slovic, "Perception of risk," Science, vol. 236, no. 4799, pp. 280-285, 1987.

[8] M. S. Horswill and F. P. McKenna, "The effect of perceived control on risk taking," Journal of Applied Social Psychology, vol. 29, no. 2, pp. 377-391, 1999 .

[9] C. T. F. Klein and M. Helweg-Larsen, "Perceived control and the optimistic bias: a meta-analytic review," Psychology and health, vol. 17, no. 4, pp. 437-446, 2002.

[10] J. Costa-Font, E. Mossialos, and C. Rudisill, "Optimism and the perception of new risks," Journal of risk research, vol. 12, no. 1, pp. 27-41, 2009.

[11] B. Lazzerini and F. Pistolesi, "Profiling risk sensibility through association rules," Expert Systems with Applications, vol. 40, no. 5, pp. 1484 1490, 2013.

[12] B. Lazzerini and F. Pistolesi, "Classifying workers into risk sensibility profiles: a neural network approach," in 8th European Modelling Symposium, 2014, pp. 33-38.

[13] S. Bandini, S. Manzoni, and F. Sartori, "Case-based reasoning to support work and learning in small and medium enterprises," in 21st IEEE Int. Conf. on Tools with Artificial Intelligence, 2009, pp. 253-260.

[14] I. C. Garcia, C. A. C. Coello, and A. Arias-Montano, "Mopsohv: A new hypervolume-based multi-objective particle swarm optimizer," in IEEE Congress on Evolutionary Computation, 2014, pp. 266-273.

[15] K. Y. Ching-Lai Hwang, Multiple attribute decision making. SpringerVerlag, 1981.

[16] K. Deb and D. Kalyanmoy, Multi-Objective Optimization Using Evolutionary Algorithms. John Wiley \& Sons, Inc., 2001.

[17] H. Ishibuchi, N. Tsukamoto, and Y. Nojima, "Evolutionary manyobjective optimization: A short review," in IEEE Congress on Evolutionary Computation, 2008, pp. 2419-2426.

[18] J. Kennedy and R. Eberhart, "Particle swarm optimization," in IEEE Int. Conf. on Neural Networks, vol. 4, 1995, pp. 1942-1948.

[19] C. A. C. Coello, G. T. Pulido, and M. S. Lechuga, "Handling multiple objectives with particle swarm optimization," IEEE Transactions on Evolutionary Computation, vol. 8, no. 3, pp. 256-279, 2004.

[20] M. Cococcioni, B. Lazzerini, and F. Pistolesi, "A semi-supervised learning-aided evolutionary approach to occupational safety improvement," in IEEE Congress on Evolutionary Computation, 2016. 